\title{
More Comprehensive Computational Number Theory
}

\section{Tianxin Cai*}

University of Zhejiang, Department of Mathematics, Hangzhou, China

Computational number theory, also known as algorithmic number theory, is the study of algorithms for performing number theoretic computations. The best known problem in the field is the integer factorization, the great common divisor or the least common multiple. The largest known primes, which are usually Mersenne primes, and they were usually found by GIMPS, the Great Internet Mersenne Prime Search.

But in my opinion, the concepts of computational number theory could be more comprehensive, covering a wide range, including all the study of number theory by computers. For me, computers are so important for number theorists, just like telescopes for astronomers.

For example, recently, I found that except 2, 5 and 11, every positive prime can be expressed as a sum of three positive integers $a, b, c$, the product abc is a cube [1]. For instance, $3=1+1+1,7=1+2+4,13=$ $1+3+9,17=1+8+8,19=4+6+9$ and $1 \times 1 \times 1=1^{3}, 1 \times 2 \times 4=2^{3}, 1 \times 3 \times 9=3^{3}$, $1 \times 8 \times 8=4^{3}, 4 \times 6 \times 9=6^{3}$.

By using a computer, we have tested that it is true for primes less than 10000. So we make the above conjecture that Except for $1 ; 2 ; 4 ; 5$;
$8 ; 11 ; 16 ; 22 ; 32 ; 44 ; 88 ; 176$, every positive integer can be expressed as a sum of three positive integers where the product of those integers is a cube.

Similarly, we conjecture that Except for $1 ; 2 ; 3 ; 5 ; 6 ; 7 ; 11 ; 13 ; 14$; $15 ; 17 ; 22 ; 23$, every positive integer can be expressed as a sum of four positive integers where the product of those integers is a $4^{\text {th }}$ power.

Open Access is a new way for us to publish and read research papers; it's easy and quick, especially in the present time of world economy crisis. I believe that all of the mathematicians, particular in developing countries and small company will benefit for it, since they might not offer the expensive fees if journal subscriptions. Therefore, I recommend that the OMICS' Applied \& Computational Mathematics Open Access policy (see http://www.omicsonline.org/OpenAccess. php for details) and the special features that the publisher provides (see http://www.omicsonline.org/special-features.php).

\section{References}

1. Cai T, Chen D. A new kind of Hilbert-Waring's problem. Math Comp. 